Vietnam Academy of Science and Technology
(VAST) Vietnam Journal of Earth Sciences
http://www.vjs.ac.vn/index.php/jse

\title{
TEC variations and ionospheric disturbances during the magnetic storm in March 2015 observed from continuous GPS data in the Southeast Asia region
}

\author{
Le Huy Minh" ${ }^{* 1}$, Tran Thi Lan ${ }^{1}$, R. Fleury ${ }^{2}$, Le Truong Thanh¹, Nguyen Chien Thang ${ }^{1}$, \\ Nguyen Ha Thanh ${ }^{1}$ \\ ${ }^{1}$ Institute of Geophysics, Vietnam Academy of Sciences and Technology \\ ${ }^{2}$ Lab-STICC, UMR 6285 Mines-Télécom, Télécom Brest, France
}

Received 7 April 2016. Accepted 15 August 2016

\begin{abstract}
The paper presents a method for computing the ionospheric total electron content (TEC) using the combination of the phase and code measurements at the frequencies $\mathrm{f} 1$ and $\mathrm{f} 2$ of the global positioning system, and applies it to study the TEC variations and disturbances during the magnetic storm in March 2015 using GPS continuous data in the Southeast Asia region. The computation results show that the TEC values calculated by using the combination of phase and code measurements are less dispersed than the ones by using only the pseudo ranges. The magnetic storm whose the main phase was on the 17th March 2015, with the minimum value of the SYM/H index of $-223 \mathrm{nT}$ is the biggest during the 24th solar cycle. In the main phase, the crests of the equatorial ionization anomaly (EIA) expanded poleward with large increases of TEC amplitudes, that provides evidence of the penetration of the magnetospheric eastward electric field into the ionosphere and of the enhancement of the plasma fountain effect associated with the upward plasma drifts. In the first day of the recovery phase, due to the effect of the ionospheric disturbance dynamo, the amplitude of northern crest decreased an amount of about $25 \%$ with respect to an undisturbed day, and this crest moved equatorward a distance of about $11^{\circ}$, meanwhile the southern crest disappeared completely. In the main phase the ionospheric disturbances (scintillations) developed weakly, meanwhile in the first day of the recovery phase, they were inhibited nearly completely. During the storm time, in some days with low magnetic activity (Ap< 50 nT), the ionospheric disturbances in the local night-time were quite strong. The strong disturbance regions with ROTI $>0.5$ concentrated near the crests of the EIA. The latitudinal-temporal TEC disturbance maps in these nights have been established. The morphology of these maps shows that the TEC disturbances are due to the medium-scale travelling ionospheric disturbances (MSTID) generated by acoustic-gravity waves in the northern crest region of the EIA after sunset moving equatorward with the velocity of about $210 \mathrm{~m} / \mathrm{s}$.
\end{abstract}

Keywords: Total electron content (TEC), equatorial ionization anomaly (EIA), medium-scale traveling ionospheric disturbance (MSTID).

(C2016 Vietnam Academy of Science and Technology

\section{Introduction}

In the middle of March 2015, the biggest magnetic storm during the 24th solar cycle

*Corresponding author, Email: 1hminhigp@ gmail.com occurred with the value of the SYM/H index of $-223 \mathrm{nT}$. The main phase of the storm was on 17 March, so it was called the Saint Patrick's Day storm. The storm is caused by the outbreak of chromosphere-type X, the 
extremely strong one, which is derived from a black line in the active zone named AR12297, observed on 11 March. According to the scientists of the Space Weather Prediction Center (SWPC), the storm can lead to the disruption of high-frequency radio transmission for hours in several large areas. It is known that during the time of magnetic storm, the ionospheric electric field disturbances observed in the medium and low latitude regions have different timescales, strongly influence the distribution of ionospheric plasma, originate from the direct penetration of the magnetospheric electric field into the ionosphere (Nishida, 1968; Vasiliunas, 1970, 1972; Jaggi \& Wolf, 1973; Fejer et al., 1979, 1990; Gonzales et al., 1979; Kelley et al., 1979, Spiro et al., 1988; Peymirat \& Fontaine, 1994; Fejer \& Scherliess, 1995; Foster \& Rich, 1997; Kikuchi et al, 2000; Kelley et al., 2003; Fejer $\&$ Emmert, 2003) and the effects of ionospheric disturbance dynamo last longer (Blanc \& Richmond, 1980; Spiro et al., 1988; Sastri, 1988; Fejer \& Scherliess, 1995; FullerRowell et al., 2002; Richmond et al., 2003). In the storm time, the basic elements of ionospheric effects in low latitude regions are generated by the morphological change of the equatorial ionization anomaly, EIA, (Appleton, 1946). During the storm, the ionospheric disturbances can appear in the night-time due to the traveling ionospheric disturbances (TIDs) that are the waveform disturbances of ionospheric plasma (Afraimovich et al., 2013; Hines, 1960). There are two types of TID having almost periodic oscillations (Georges, 1968): large-scale TID (LSTID) characterized by high velocity (> $300 \mathrm{~m} / \mathrm{s}$ ) and long cycle (> 1h) and mediumscale TID (MSTID) characterized by lower speed $(50-300 \mathrm{~m} / \mathrm{s})$ and shorter cycle $(10 \mathrm{~min}$ to $1 \mathrm{~h})$. LSTIDs appear as a chain of shortwave with the small number of cycles, meanwhile, MSTIDs can have several cycles (Francis, 1974). In addition to the mentioned TIDs, there are MSTIDs having no cycle that appear as the oscillations with different cycles of the electron density. MSTIDs are present in the F region of the ionosphere, whereas LSTIDs are much scarcer, only appear in case of the big magnetic storms. LSTIDs originate from the auroral region (Georges, 1968; Davis, 1971) while the observations of MSTIDs suggest that their source mechanisms are in the lower latitude regions (Munro, 1958; Davies \& Jones, 1971). Many studies on TID based on observation of the ionospheric total electron content (TEC) from the dense network of GPS stations in Japan (Saito et al., 1998; Shiokawa et al., 2002; Afraimovich et al., 2009), in North America (Tsugawa et al., 2007), in Europe (Borries et al., 2009), and from the chain of GPS stations in the region of AfricaEurope Shimeis et al. (2015) have also observed the signs of TID in the medium and low latitude regions. This paper presents the observation results of TEC variations and ionospheric disturbances from GPS data in Vietnam and the Southeast Asia region during the magnetic storm occurring from 15 March to 28 March 2015.

\section{Data and calculation method}

Data used in this paper are from the continuous GPS stations in Vietnam and the Southeast Asia region, whose names, magnetic coordinates and latitudes are listed in Table 1 and presented in Figure 1. From XMIS to PHUT the latitudes change from $19.58^{\circ}$ to $14.89^{\circ}$, so that we can obtain information about the equatorial ionization anomaly in the Southeast Asia region (Le Huy et al., 2014). Among these 8 stations, PHUT and HUE2 stations with GSV4004 receiver can provide the $\mathrm{S}_{4}$ indices, the standard deviation of the code/carrier phase $\left(\sigma_{\mathrm{ccd}}\right)$, the specific parameters of the amplitude scintillation of GPS signals when traveling through the ionosphere.

To calculate TEC, a method of using the pseudo range measurements is presented in (Le Huy et al., 2014; Le Huy Minh et al., 2006), in this paper we introduce the method of using the combination of the phase and pseudo range measurements. 


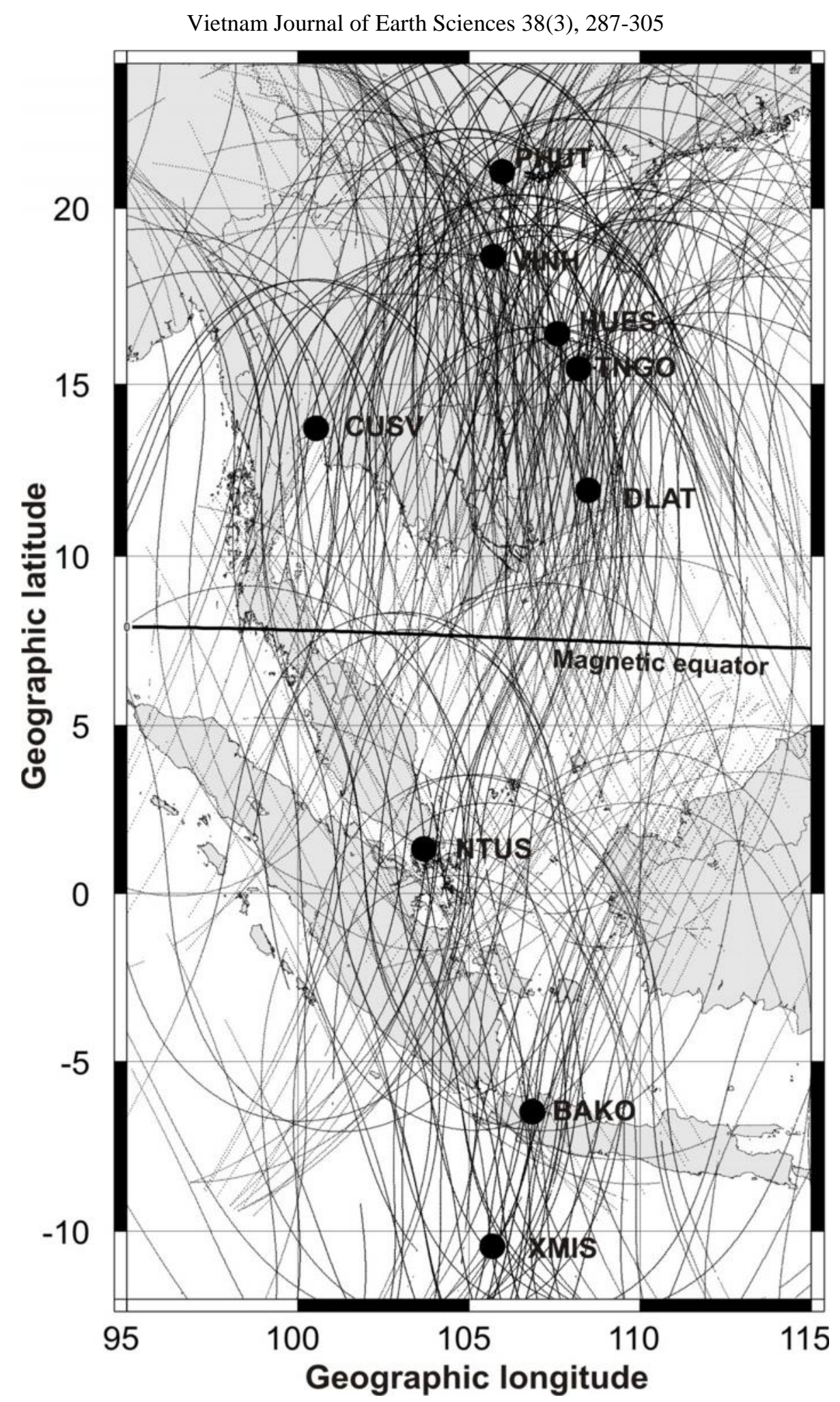

Figure 1. Location of GPS receivers and traces of the visible satellites at 400km altitudes on the 15 March 2015 
L.H. Minh, et al./Vietnam Journal of Earth Sciences 38 (2016)

$\underline{\text { Table 1. GPS stations in Vietnam and Southeast Asian region }}$

\begin{tabular}{|c|c|c|c|c|c|}
\hline \multirow{2}{*}{ No } & \multirow{2}{*}{ Station } & \multirow{2}{*}{ Receiver } & \multicolumn{2}{|c|}{ Geographic coordinate } & \multirow{2}{*}{$\begin{array}{c}\text { Magnetic Latitude } \\
(2015)\end{array}$} \\
\hline & & & Latitude & Longitude & \\
\hline 1 & PHUT & GSV4004 & 21.02938 & 105.95872 & 14.89 \\
\hline 2 & VINH & CORS5700 & 18.64999 & 105.69659 & 12.32 \\
\hline 3 & HUES & GSV4004 & 16.45883 & 107.59346 & 9.58 \\
\hline 4 & TNGO & CORS5700 & 15.44722 & 108.20385 & 8.92 \\
\hline 5 & CUSV & NETRS & 13.73591 & 100.53392 & 6.86 \\
\hline 6 & DLAT & JAVAD & 11.94526 & 108.48173 & 5.16 \\
\hline 7 & NTUS & LEICA & 1.34580 & 103.67996 & -6.62 \\
\hline 8 & BAKO & LEICA & -6.49106 & 106.84891 & -15.10 \\
\hline 9 & XMIS & NETR9 & -10.44997 & 105.68849 & -19.58 \\
\hline
\end{tabular}

In the dual frequency GPS measurements, frequencies $f_{1}$ and $f_{2}$ are measurable, so they the pseudo range measurement $p_{k j}^{i}$ and the can be written (Liu et al., 1996; Carrano \& phase measurement $L_{k j}^{i}$ at the GPS Groves, 2009):

$$
\begin{aligned}
& p_{1 j}^{i}=s_{0 j}^{i}+d_{\text {ion } 1 j}^{i}+d_{\text {tropj }}^{i}+c\left(\tau^{i}-\tau_{j}\right)+b_{p 1}^{i}+b_{p 1 j}+m_{1}^{p}+\varepsilon_{1}^{p} \\
& p_{2 j}^{i}=s_{0 j}^{i}+d_{\text {ion } 2 j}^{i}+d_{\text {tropj }}^{i}+c\left(\tau^{i}-\tau_{j}\right)+b_{p 2}^{i}+b_{p 2 j}+m_{2}^{p}+\varepsilon_{2}^{p} \\
& L_{1 j}^{i}=s_{0 j}^{i}+d_{\text {ion } 1 j}^{i}+d_{\text {tropj }}^{i}+c\left(\tau^{i}-\tau_{j}\right)+b_{\phi 1}^{i}+\lambda_{1} N_{1 j}^{i}+m_{1}^{\phi}+\varepsilon_{1}^{\phi} \\
& L_{2 j}^{i}=s_{0 j}^{i}+d_{\text {ion } 2 j}^{i}+d_{\text {tropj }}^{i}+c\left(\tau^{i}-\tau_{j}\right)+b_{\phi 2}^{i}+\lambda_{2} N_{2 j}^{i}+m_{2}^{\phi}+\varepsilon_{2}^{\phi}
\end{aligned}
$$

where $i$ and $j$ indices are the satellite $i$ and the receiver $\mathrm{j}$ respectively; $\mathrm{s}_{0}$ is the real distance between the receiver and the satellite, $d_{\text {ion }}$ and $\mathrm{d}_{\text {trop }}$ are the ionospheric delay and the tropospheric delay, $\mathrm{c}$ is the speed of light in vacuum, $\tau$ is the satellite clock error or the receiver clock error, $\mathrm{b}$ is the device delay of the satellite or of the receiver, $\mathrm{N}$ is the multivalued integer, $\lambda$ is the transmission wavelength, $m$ is the multipath effect in the pseudo range measurements or in the phase measurements, $\varepsilon$ is the interference in the corresponding measurements at the frequencies $\mathrm{f}_{1}$ and $\mathrm{f}_{2}$.

According to the Appleton formula (Budden, 1985), the ionospheric delay conforming to slant total electron content
(STEC) between the $R_{x}$ receiver and the $T_{x}$ satellite can be written:

$d_{\text {ion }}=s^{\prime}-s_{0}=\int_{T_{x}}^{R_{x}}\left(\frac{1}{n}-1\right) d l=\frac{40,3}{f^{2}} \int_{T_{x}}^{R_{x}} N(l) d l=\frac{40,3}{f^{2}} \operatorname{STEC}(2)$

where s' is the apparent distance between the receiver and the satellite, $\mathrm{N}$ (1) is the electron density along the satellite-receiver line in $\mathrm{el} / \mathrm{m}^{3}, \mathrm{n}$ is the refractive index, and $\mathrm{f}$ is the frequency of radio waves in $\mathrm{Hz}$.

The ionosphere acts as the scattering medium for GPS signals, but the troposphere is the non-scattering medium, so the tropospheric delay can be eliminated by using the subtraction (1b)-(1a) and (1c)-(1d). Using the subtraction (1b)-(1a) and ignoring the multipath effect and the interference, we have:

$$
p_{2 j}^{i}-p_{1 j}^{i}=d_{i o n 2 j}^{i}-d_{i o n 1 j}^{i}+\left(b_{p 2}^{i}-b_{p 1}^{i}\right)+\left(b_{p 2 j}-b_{p 1 j}\right)=d_{i o n 2 j}^{i}-d_{i o n 1 j}^{i}+b_{p}^{i}+b_{p j}
$$


By the combination of the formulas (2) and (3) we have:

$$
\begin{gathered}
\text { STEC }=\frac{1}{40,3} \frac{f_{1}^{2} f_{2}^{2}}{f_{1}^{2}-f_{2}^{2}}\left(p_{2 j}^{i}-p_{1 j}^{i}-\left(b_{p}^{i}+b_{p j}\right)\right) \quad \text { (4) interference, we have: } \\
L_{1 j}^{i}-L_{2 j}^{i}=d_{i o n 1 j}^{i}-d_{i o n 2 j}^{i}+\left(b_{1 \phi}^{i}-b_{2 \phi}^{i}\right)+\left(b_{1 \phi j}-b_{2 \phi j}\right)+\left(\lambda_{1} N_{1 j}^{i}-\lambda_{2} N_{2 j}^{i}\right)= \\
=d_{i o n 1 j}^{i}-d_{i o n 2 j}^{i}+b_{\phi}^{i}+b_{\phi j}+\left(\lambda_{1} N_{1 j}^{i}-\lambda_{2} N_{2 j}^{i}\right)
\end{gathered}
$$

Using the subtraction (1c)-(1d) and ignoring the multipath effect and the

Combining (2) with (5) we have:

$$
S T E C=\frac{1}{40,3} \frac{f_{1}^{2} f_{2}^{2}}{f_{1}^{2}-f_{2}^{2}}\left[\left(L_{1 j}^{i}-L_{2 j}^{i}\right)-\left(b_{\phi}^{i}+b_{\phi j}\right)-\left(\lambda_{1} N_{1 j}^{i}-\lambda_{2} N_{2 j}^{i}\right)\right]
$$

In the formulas (4) and (6) STEC is calculated in TECU, 1TECU $=10^{16} \mathrm{el} / \mathrm{m}^{2}$. The vertical total electron content, VTEC or written as TEC, observed at the breakpoint of the ionosphere is determined from singlelayer model (Klobuchar, 1986):

$$
T E C=S T E C \cdot \cos \left[\arcsin \left(\frac{R \cos \theta}{R+h}\right)\right]
$$

where $\theta$ is the satellite elevation angle in degree $\left({ }^{\circ}\right), \mathrm{R}=6371.2 \mathrm{~km}$ is the average radius of the Earth, $h$ is the height of ionospheric single layer, often considered as $400 \mathrm{~km}$ (Zhao et al., 2009).

So, to work out the value of STEC from the formula (4) we need to calculate the device delays $b_{p}=b_{p}^{i}+b_{p j}$ (the constant for each pair of satellite-receiver), from the formula (6) we need to calculate the device delays $\quad b_{\phi}=b_{\phi}^{i}+b_{\phi j} \quad$ and the nondetermination of initial phase $\lambda_{1} N_{1 j}^{i}-\lambda_{2} N_{2 j}^{i}$ that are also the constants.

In the formula (4),
$\operatorname{STEC}_{p}=\frac{1}{40,3}\left(\frac{f_{1}^{2} f_{2}^{2}}{f_{1}^{2}-f_{2}^{2}}\right)\left(p_{2 j}^{i}-p_{1 j}^{i}\right)$ is a quantity that is clearly determined, however due to the influence of interference and multipath effect, its values are usually dispersed; and in the formula (6), the quantity

$$
S T E C_{\phi}=\frac{1}{40,3}\left(\frac{f_{1}^{2} f_{2}^{2}}{f_{1}^{2}-f_{2}^{2}}\right)\left(L_{1 j}^{i}-L_{2 j}^{i}\right)
$$

precisely determined but suffers the jumps due to the cycle slip (Carrano \& Groves, 2009). We use the quantity $\mathrm{STEC}_{\mathrm{p}}$ to eliminate the jumps in the $\mathrm{STEC}_{\phi}$ as follows. Within each continuous distance of the satellite tracks, STEC $_{p}$ is approximated by the fourth-degree polynomial. The quantity $\mathrm{STEC}_{\phi}$ is compared with $\mathrm{STEC}_{\mathrm{p}}$, which is smoothed by polynomial approximation, to evaluate the magnitude of the jumps in $\mathrm{STEC}_{\phi}$ on the same satellite track. $\mathrm{VTEC}_{\phi}$ in case of regulating the jumps is calculated and compared with the value of VTEC from the global TEC model (CODG model) at the corresponding time in order to determine the total delay of device delay and the nondetermination of initial phase that is similar to the estimation of device delay in calculating the absolute TEC by using the pseudorange measurements. The value of total delay for each pair of satellite-receiver in the observation day is the average value of total delay at each observation time. To reduce multipath effect in the low satellite elevation angles, the values of TEC used to compare with TEC from the global model are often chosen in accordance with the satellite elevation angle $\alpha \geq 30^{\circ}$.

To study the ionospheric scintillation from data of the receiver GSV4004, we use the amplitude scintillation index $\mathrm{S}_{4}$ that is calculated according to the formula (Van Dierendonck et al., 1993):

$$
S_{4}=\sqrt{S_{4 t o t}^{2}-S_{4 c o r}^{4}}
$$


where $S_{4 \text { tot }}$ is the total $S_{4}$ and $S_{4 \text { cor }}$ is the corrected $S_{4}$ due to the interference effect. Both of these quantities are obtained directly from the output signal of the receiver GSV4004. $\mathrm{S}_{4}$ obtained in such way contains the multipath effect, especially in low satellite elevation angle, therefore the scientists often rely on the parameter $\sigma_{\mathrm{ccd}}$, which characterizes the influence of multipath effect, to establish a filter limit for each station (Tran Thi Lan et al., 2011; Abadi et al., 2014; Tran Thi Lan et al., 2015). The method is based on selecting days of quiet ionosphere in each year at each station, graphing the relationship between the parameter $\sigma_{c c d}$ and the index $S_{4}$, finding a line to separate the scintillation due to multipath effect from the one due to the ionosphere, then the $\mathrm{S}_{4}$ indices over this line are supposed to be caused by multipath effect, and the ones under this line are supposed to be caused by ionospheric effect. Applying such filter limit on days of any data at each station, we obtain the index $\mathrm{S}_{4}$ caused by the ionospheric scintillation. The index $\mathrm{S}_{4}$ obtained in such way is $S_{4}$ for the different satellite elevation angles, to get the vertical $S_{4}$, we apply the formula (Spogli et al., 2009):

$$
S_{4}\left(\alpha=90^{\circ}\right)=S_{4}(\alpha) \sin ^{b}(\alpha)
$$

where $\alpha$ is the satellite elevation angle, $b$ is chosen to be 0.9 .

Another index indicating the level of ionospheric disturbance - ROT, which is the rate of change of TEC with respect to time calculated from the L1 and L2 phase measurements, is used (Pi et al., 1997):

$$
R O T=\frac{V T E C_{u}^{k}-V T E C_{u-1}^{k}}{t_{u}-t_{u-1}}
$$

where $\mathrm{k}$ is the visible satellite, $\mathrm{u}$ is the time of observation and ROT is calculated in TECU/minute. The measurements of ROT point out the small-scale variations on the background of a larger-scale trend. The rate of TEC index, ROTI, is defined as the standard deviation of ROT at 5-minute interval:

$$
R O T I=\sqrt{\left\langle R O T^{2}\right\rangle-\langle R O T\rangle^{2}}
$$

Ordinarily, ROTI $\geq 0.5$ reveals the presence of ionospheric anomalies on the scale of a few kilometers or more (Ma \& Maruyama, 2006).

\section{Calculation results and discussion}

\subsection{Magnetic parameters during storm time}

Figure 2 represents the component $X$ of the solar wind $\mathrm{V}_{\mathrm{x}}$, the component $\mathrm{Z}$ of the interplanetary magnetic fields $B_{z}$, the symmetric disturbance field in $\mathrm{H}$ index $\mathrm{SYM} / \mathrm{H}$ and the auroral electrojet index $\mathrm{AE}$ between 15 March and 28 March 2015, in which $V_{x}$ and $B_{z}$ are moving-averaged in the period of an hour. It is necessary to note that the time in each day of the dataset is based on the universal time (UT), the local time LT equals the UT plus 7 , in the figure there are two vertical lines corresponding to the start times of the main phase and the recovery phase of the storm examined. At 18:00 UT on 15 March $V_{x}$ began to increase from $295 \mathrm{~km} / \mathrm{s}$ and reached a maximum of about $690 \mathrm{~km} / \mathrm{s}$ at the end of 18 March. $V_{x}$ ranged between 550 $\mathrm{km} / \mathrm{s}$ and $690 \mathrm{~km} / \mathrm{s}$ from 18 to $25 \mathrm{March}$; in three following continuous days of 26-28 March $\mathrm{V}_{\mathrm{x}}$ decreased from $550 \mathrm{~km} / \mathrm{s}$ to 400 $\mathrm{km} / \mathrm{s}$. In the period of 15-28 March, except for March 17, $\mathrm{B}_{\mathrm{z}}$ varied from $-7 \mathrm{nT}$ to $\sim 11 \mathrm{nT}$. On 17 March $B_{z}$ unexpectedly changed from $\sim 8$ $\mathrm{nT}$ at 3:17 UT to $21.6 \mathrm{nT}$ at 4:34 UT; then $\mathrm{B}_{\mathrm{z}}$ suddenly reduced from positive value to negative value, which was essentially the movement of $\mathrm{B}_{\mathrm{z}}$ from the northward direction to the southward direction; and in most of time between 4:43 UT and 23:12 UT B $\mathrm{B}_{\mathrm{z}}$ was toward the South; but in 2 periods of 6:09 UT - 6:33 UT and 8:49 UT - 11:27 UT, $B_{z}$ was toward the North. The index Dst demonstrates that the main phase occurred on 17 March from 5:00 UT to $23: 00 \mathrm{UT}$; the minimum value of SYM/H index of $-223 \mathrm{nT}$ indicates that it was the big storm. The recovery phase started after the main phase from 23:00 UT; the $\mathrm{SYM} / \mathrm{H}$ index began to increase in accordance with the movement of $B_{z}$ from the 
Vietnam Journal of Earth Sciences 38(3), 287-305

South to the North. The variations of SYM/H index show that until the end of 28 March the value of SYM/H index almost came back to that on 15 March, thus the recovery phase of this storm completed at the end of 28 March. In the main phase of the storm, the $\mathrm{AE}$ index rose to a peak of $1570 \mathrm{nT}$; between 18 March and 28 March, the maximum of AE index was from $1130 \mathrm{nT}$ on 19 March to $408 \mathrm{nT}$ on 27 March. In the main phase of the storm, the magnetic activity index Ap reached the maximum of $179 \mathrm{nT}, 80 \mathrm{nT}, 94 \mathrm{nT}$ on 17, 18 and 22 March respectively; and on other days, the Ap index was smaller than $50 \mathrm{nT}$.

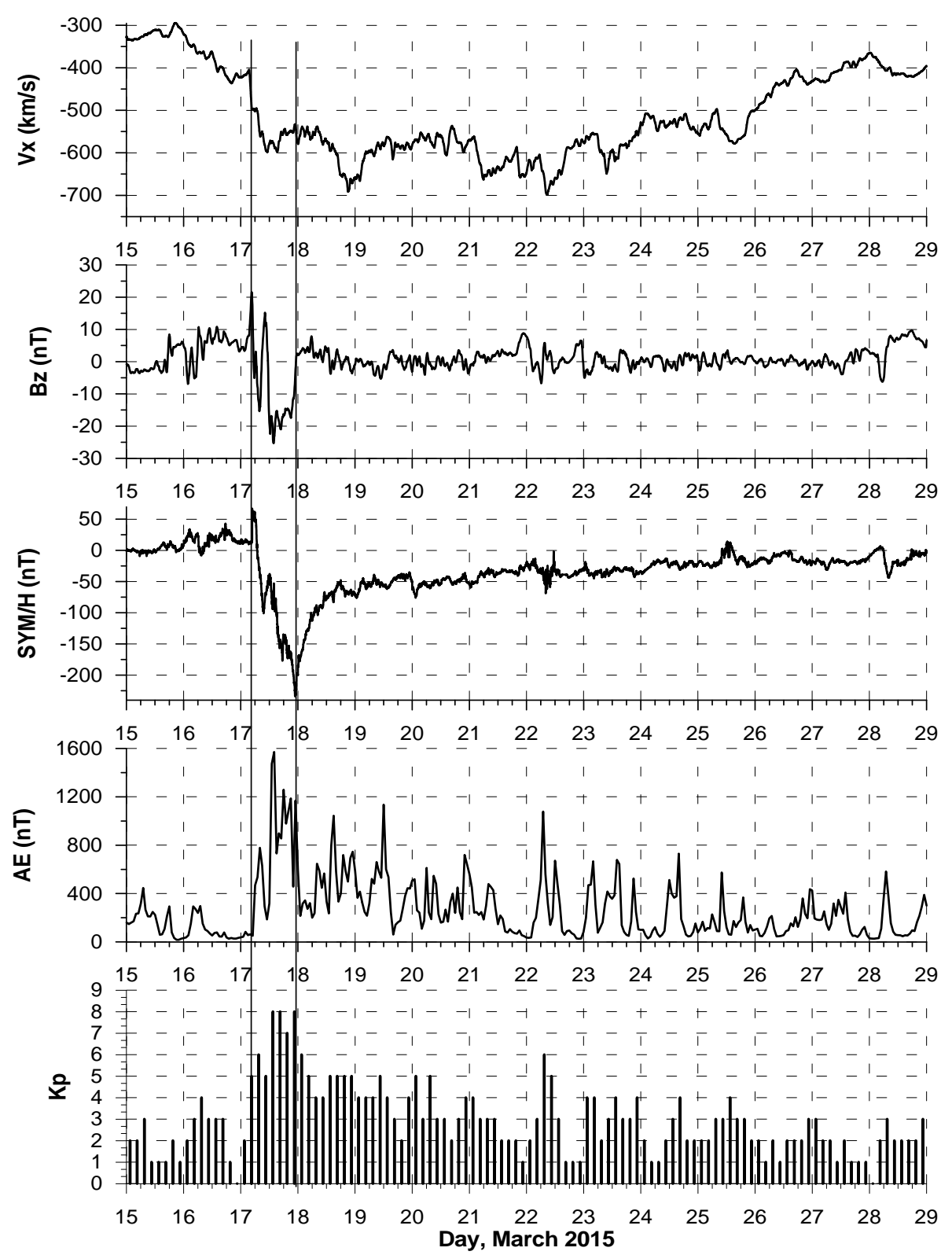

Figure 2. From top to bottom, $X$-component of solar wind speed $\left(V_{x}\right)$, z-component of the $\mathrm{IMF}\left(\mathrm{B}_{\mathrm{z}}\right)$, symmetric disturbance field in $\mathrm{H}$ index $(\mathrm{SYM} / \mathrm{H})$, auroral magnetic index (AE) and planetary $\mathrm{Kp}$ are displayed. The main phase of the storm is limited in two vertical solid lines 


\subsection{TEC variations}

To compare the calculation result of TEC from the pseudo range measurements and that from the combination of the phase and pseudo range measurements as mentioned above, Figure 3 presents the computation results of TEC by both methods for data at Phu Thuy GPS station on 1 January 2012. It can be seen that the shapes of the TEC curves calculated from both types of data are identical. However it is obvious that on each satellite line the values of TEC obtained from the method presented here are less dispersed. It indicates that the values of TEC computed by using the combination of phase and pseudo range measurements are more reliable than those by using the pseudo range measurements, as some other authors in the world have noticed (Liu et al., 1996, Carrano \& Groves, 2009). The calculation method of TEC presented above is applied to the dataset of GPS stations in the Southeast Asia region in the period from 15 to 28 March 2015.
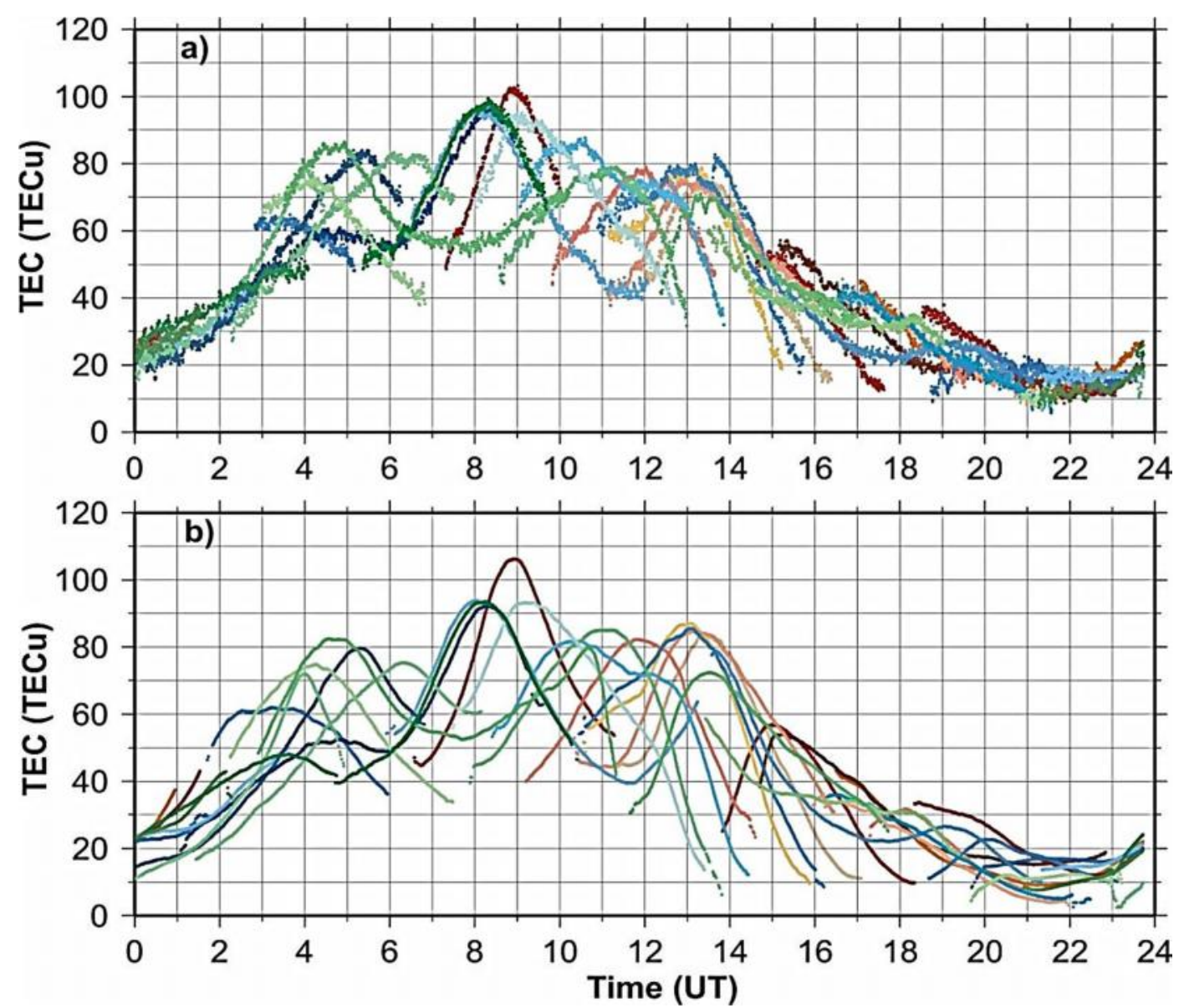

Figure 3. Total electron content on the 15 March 2015 computed a) by using pseudorange measurements, and b) by using the combination of carrier phase and pseudorange measurements

Figure 4 presents the temporal-latitudinal maps of TEC in the Southeast Asia region between 15 and 28 March 2015. In Figure 4 the location of the magnetic equator is indicated by the line in the latitude of $7-8^{\circ} \mathrm{N}$.
The maps in Figure 4 clearly shows the structure of the equatorial ionization anomaly in the Southeast Asia region, including a crest in the northern hemisphere and another in the southern hemisphere that is almost 
symmetrical to each other over the magnetic equator. The morphology of anomaly changed continuously day by day during the storm. Figure 5 presents the amplitude, appearance time and latitude of the corresponding anomaly crest in that period. The amplitudes of anomaly on 16 and 17 March rose markedly, the crest expanded poleward and the appearance time was earlier than that on 15 March. On 18 March, the beginning day of the recovery phase, the anomaly degenerated, only the northern crest existed with the amplitude decreasing remarkably (about $25 \%$ ), it moved equatorward a distance of $11^{\circ}$ compared to that on 17 March and its appearance time was a few hours earlier than that on 19 and 17 March, meanwhile the southern crest completely disappeared. The complete disappearance of the southern crest of the equatorial ionization anomaly was also observed by Lin et al. (2005) in the big magnetic storm within September-October 2003. In the first phase of the magnetic storm, the vertical component of the interplanetary magnetic field $\mathrm{B}_{\mathrm{z}}<0$, the interactions between the solar wind and the southward interplanetary magnetic field cause the eastward electric field to penetrate directly into the ionosphere (for example, Nishida, 1968; Kikuchi et al., 2000; Fejer \& Emmert, 2003). This eastward electric field increases the fountain effect as well as the amplitude of anomaly crest and promotes the poleward expansion of the anomaly crest. In the storm when the high-energy particle flow of the solar wind deeply penetrates into the polar atmosphere and heats it, there is the appearance of the meridian neutral wind blowing equatorward. The complex interactions between the neutral wind and the Earth's magnetic field cause the phenomenon called the ionospheric disturbance dynamo (Blanc \& Richmond, 1980) in which the electric field in the low latitude region is in the westward direction, in contrast to the eastward electric field in normal condition. This westward parallel electric field appears in the recovery phase, causing the downward plasma drift, the decrease in the fountain effect and the degeneration of the structure of the equatorial ionization anomaly.
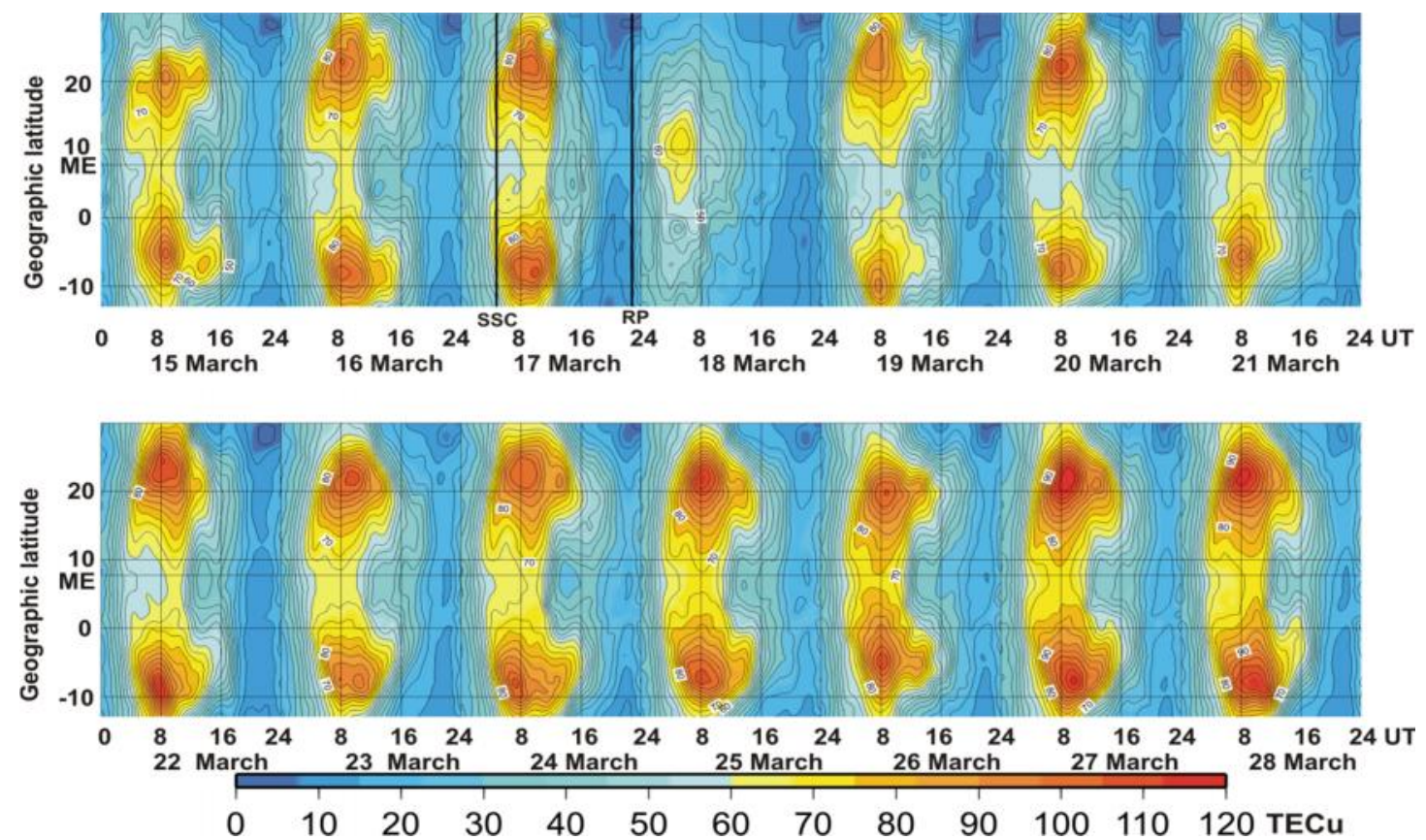

Figure 4. Time and latitudinal TEC maps for the period between 15 and 28 March 2015. Contour interval: 5TECu. SSC: sudden commencement of the storm, RP: the beginning of the recovery phase 
L.H. Minh, et al./Vietnam Journal of Earth Sciences 38 (2016)
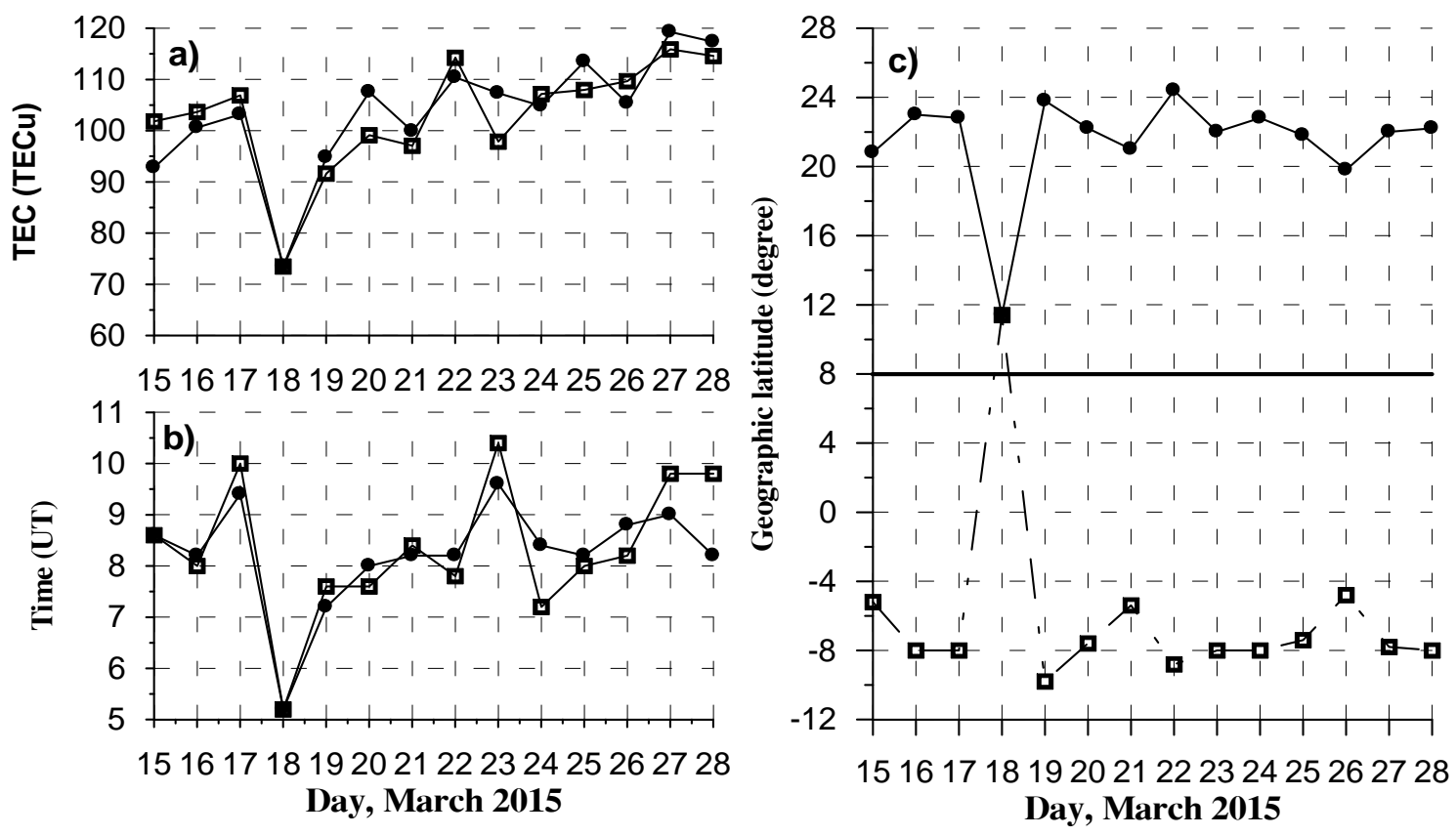

Figure 5. a) Maximum TEC, b) appearance time and c) latitude of the northern (black cycle) and southern (open rectangular) EIA crests from 15 to 28 March 2015

\subsection{Ionospheric disturbances}

Figure 6 shows the variations of ROTI $\geq$ 0.5 at Hue station and ROTI $\searrow .575$ at Phu Thuy station (ROTI below this level appears in almost all the observation times, and such ROTI index does not reflect the disturbances in the ionosphere), and the $\mathrm{S}_{4}$ indices selected and calculated as presented above at Phu Thuy and Hue stations from 15 to 28 March 2015 . Figure 7 indicates ROTI $\searrow 0.5$ at TNGO, CUSV, DLAT, NTUS, BAKO and XMIS stations in that period. Figure 6 demonstrates the definite correlation between the amplitude scintillation index $\mathrm{S}_{4}$ and the index ROTI calculated from the total electron content obtained from the phase measurements, although the numerical values of these indices are different. These indices almost appear at the night-time from 12:00 UT to 18:00 UT (i.e. from 19:00 LT to 01:00 LT of the following day). In the period studied, on 16 , 19, 24-28 March the extremely strong ionospheric disturbances were observed at both stations, on 17, 18, 20-25 March very few ionospheric disturbances were observed at both stations, on 15 and 25 March the ionospheric disturbances observed at Hue stations were much more than those at PHUT station. The distance between HUE and PHUT is about $500 \mathrm{~km}$, the ionospheric anomalies at two stations have the same and different characteristics that indicate the spatial scales of the ionospheric anomalies are not identical on the different days. We also observe a similar condition in Figure 7 . The ROTI indices at five stations (TNGO, CUSV, DLAT, NTUS and BAKO) on 16, 19, 24-28 March show that the ionospheric disturbances observed at these stations were obvious. In XMIS station, the furthest station from the equator in the southern hemisphere, the ionospheric disturbances observed were rather plenty on 16 and 26 March as in other stations, on other days the ionospheric disturbances were also observed but ROTI $\searrow 0.5$ rarely appeared. In all eight stations during the night of 18 March, the 
ionospheric disturbances hardly appeared. As mentioned above, on 18 March the ionospheric disturbance dynamo developed, the EIA degenerated, the southern crest of the anomaly disappeared, the northern crest moved equatorward a distance of about $11^{\circ}$ compared to that on $17 \mathrm{March}$; therefore, when the ionospheric disturbance dynamo developed, the westward electric field was enhanced, causing the ionospheric F-layer to move downward and the ionospheric disturbances to be prevented. On 17 March, the developing day of the main phase, due to the direct penetration of the magnetospheric eastward electric field into the ionosphere, the equatorial ionization anomaly was enhanced; the amplitude of the crest increased compared to that on 16 March; the crest expanded poleward; the appearance time of the maximum was later; however, it can be seen in Figure 6 and Figure 7 that the ionospheric disturbances in the night of 17 March poorly or hardly developed. On 16, 19, 24-28 March the appearance of the values ROTI $\searrow 0.5$ were almost observed in all the stations, the magnetic activity index Ap reached $48 \mathrm{nT}$ on 19 March and was less than or equal to $22 \mathrm{nT}$ on other days, so on the appearance days of the ionospheric disturbances; the magnetic activity was relatively weak. To study the spatial distribution of the ionospheric disturbances, Figure 8 indicates the distribution of the ROTI indices observed in the night-time of 16 and 19 March, a day before the main phase and a day in the recovery phase of the storm, Figure 9 shows the latitudinal distribution of the indices ROTI $\searrow .5$ on these two days. On 16 and 19 March the northern and southern crests were in the latitudes of $22.4^{\circ} \mathrm{N}, 23.4^{\circ} \mathrm{N}$ and $8.0^{\circ} \mathrm{S}$, $10.0^{\circ} \mathrm{S}$ respectively, the location of the equator was in the latitude of about $7.8^{\circ} \mathrm{N}$. Accordingly, Figure 8 and Figure 9 demonstrate that the indices ROTI $\searrow 0.5$ mostly concentrated in the latitude of $16^{\circ} \mathrm{N}$ and $6^{\circ} \mathrm{S}$, the location of the maximum disturbances (at night) concentrated closer to the equator than the crest of the equatorial ionization anomaly did (by day).

To examine the effects of TEC disturbances, we determine the components of TEC disturbances according to the formula:

$$
\triangle T E C=T E C-T E C_{t b 2 h}
$$

where $\mathrm{TEC}_{t b 2 h}$ is the value of TEC in the corresponding time that is moving-averaged in the period of two hours, this process has been used by many authors in order to analyze the medium-scale and large-scale TIDs (Saito et al., 1998; Borris et al., 2009). In some cases when the disturbances have the shorter cycles than we choose, the period of the moving average is 30 minutes (Figure 10) or 1 hour. The separation of the disturbance components is executed for each satellite, then the temporal-latitudinal maps of TEC disturbances are established by the same process as those of TEC. The results of establishing TEC disturbance maps in the night-time of 16, 19, 24-28 March 2015 are presented in Figure 11.

The TEC disturbances maps are established for the period from 12:00 UT to 24:00 UT (from 19:00 LT to 07:00 LT of the next day) because the disturbances mainly appear in this period (Figure 6 and Figure 7). Although the amplitude of TEC disturbances is small compared to that of TEC, but the obtainment of the TEC disturbance maps having the almost unified characteristics in Figure 11 allows affirming that the process of obtaining TEC disturbances as presented above is logical. The TEC disturbance maps in Figure 11 have the similar forms, two positive disturbance regions are at two sides of the magnetic equator, the negative disturbances are in the magnetic equator and adjacent regions, the negative disturbance region near the magnetic equator in the south is larger than that in the north. In the period from 20:00 UT to 24:00 UT (03:00 LT-07:00 LT) the negative disturbances are mostly in 
L.H. Minh, et al./Vietnam Journal of Earth Sciences 38 (2016)

the latitude region of $8^{\circ} \mathrm{S}-24^{\circ} \mathrm{N}$. Figure 4 shows that on the TEC map the equatorial ionization anomaly are nearly symmetrical over the magnetic equator, meanwhile, on the TEC disturbance map (Figure 11) the symmetry of two disturbance regions at two sides of the magnetic equator is less apparent. In the north of the magnetic equator, the positive disturbance regions stretching continuously in the dashed line direction in Figure 11 give us the picture of the ionospheric disturbances moving equatorward from the northern anomaly crest region. With the scale of about 200-300 km as in Figure 11, these disturbances can be considered the medium-scale traveling ionospheric disturbances (MSTID). Furthermore, Figure 10 shows that the TEC disturbance containing several cycles with different wavelengths is also a characteristic of MSTID. If the dashed line direction in Figure 11 is considered to indicate the movement direction of MSTID, the movement velocity of the disturbances on the examined days can be calculated at about $210 \mathrm{~m} / \mathrm{s}$ toward the south. The apparent asymmetry of the TEC disturbance regions in Figure 11 suggests that maybe these MSTIDs do not move across the magnetic equator.

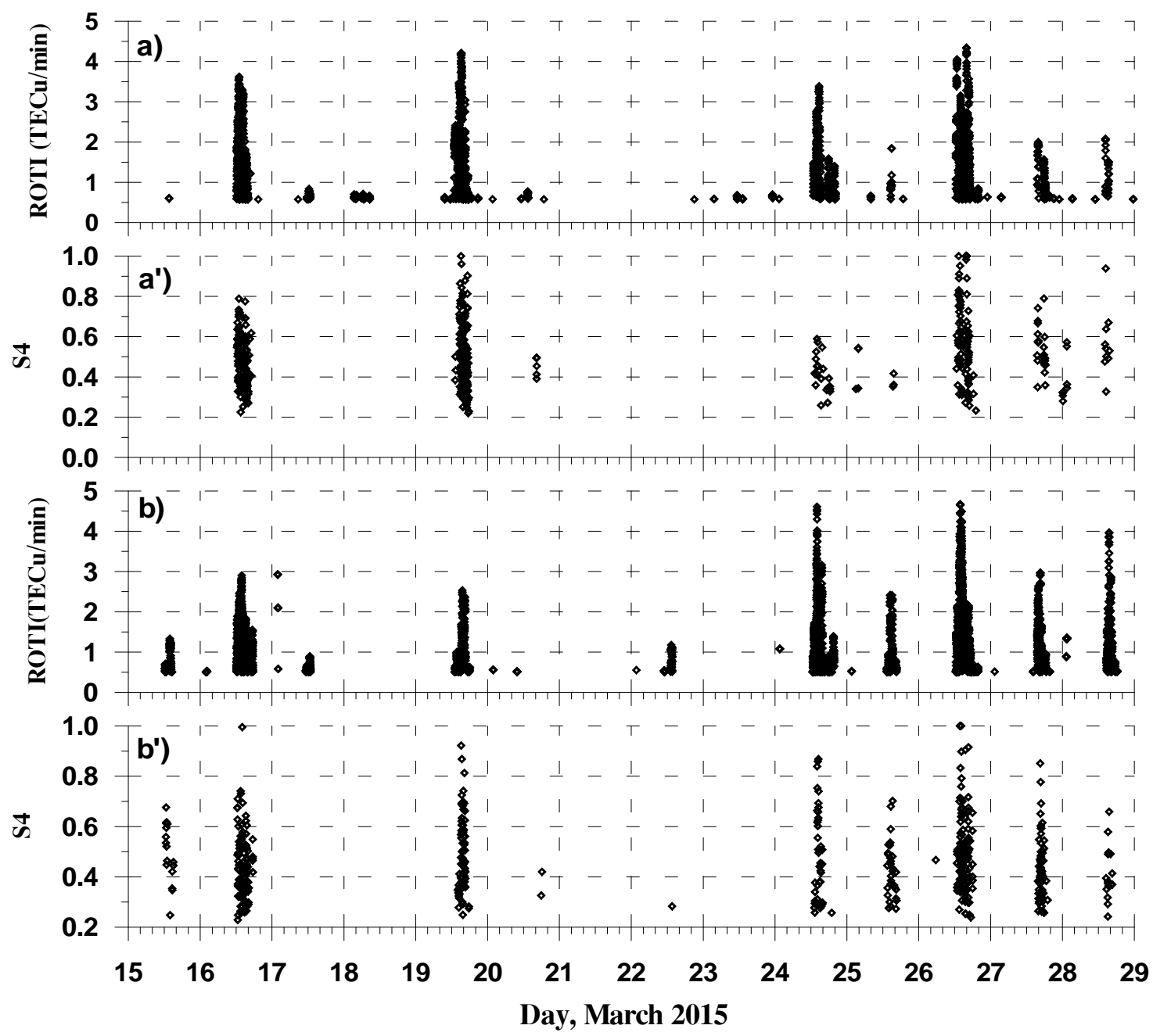

Figure 6. ROTI and $S_{4}$ indices a) and a') at PHUT and b) and b') at HUES from 15 to 28 March 2015 
Vietnam Journal of Earth Sciences 38(3), 287-305

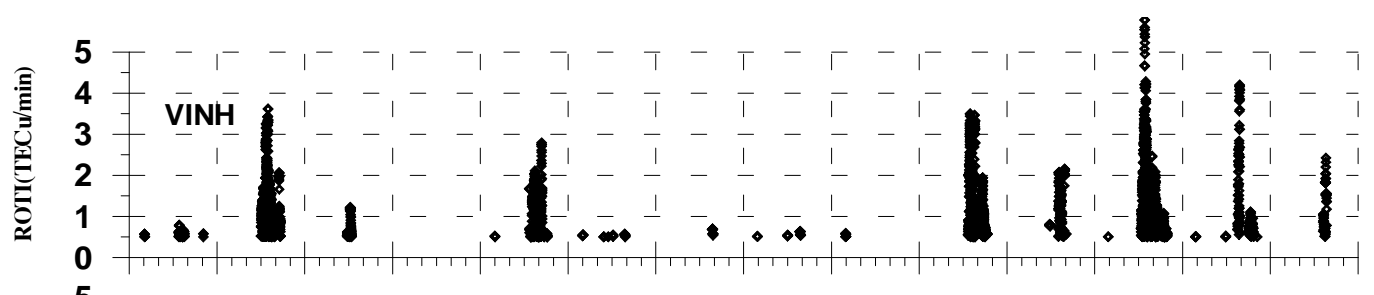

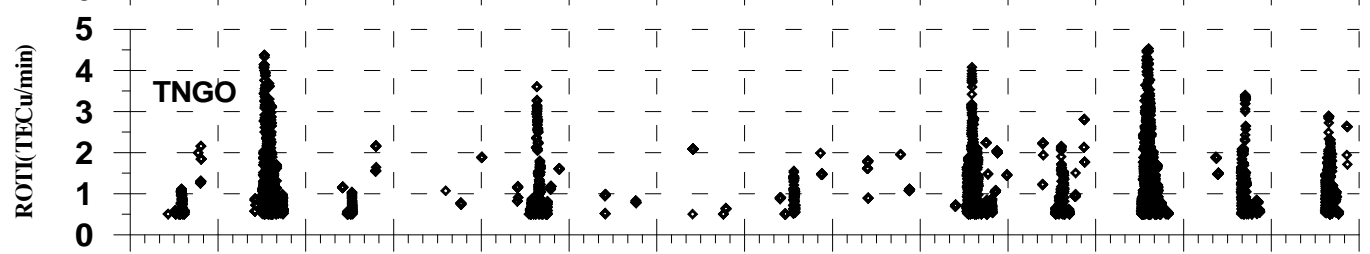

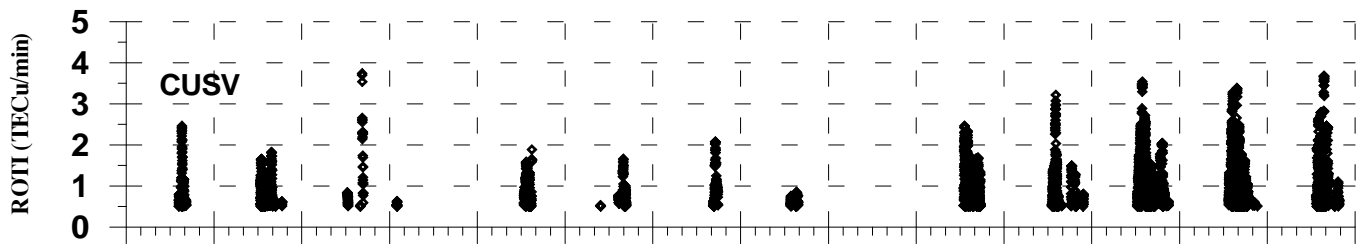
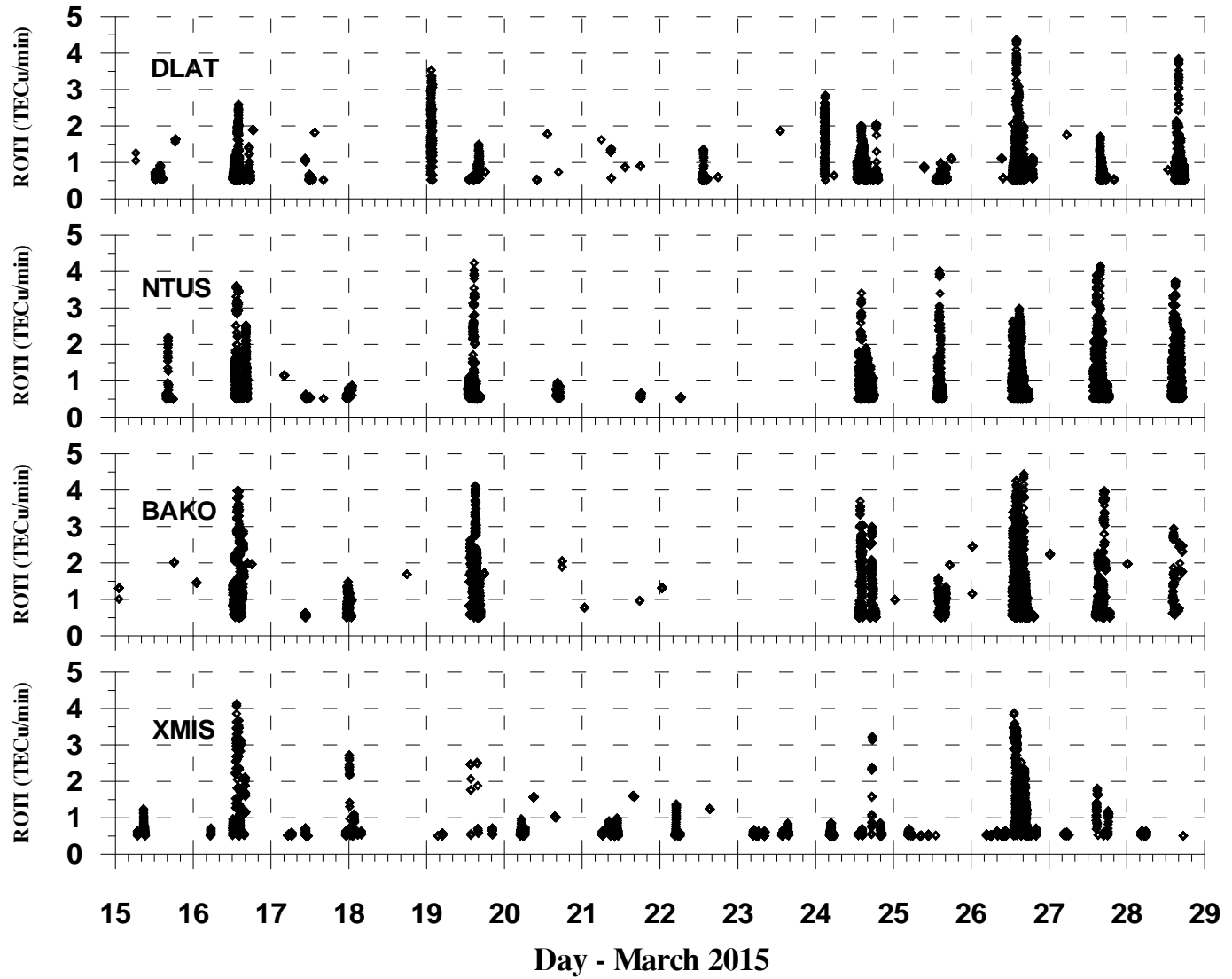

Figure 7. From top to bottle ROTI $\geq 0.5$ observed at VINH, TNGO, CUSV, DLAT, NTUS, BAKO and XMIS stations for the period 15-28 March 2015 
L.H. Minh, et al./Vietnam Journal of Earth Sciences 38 (2016)
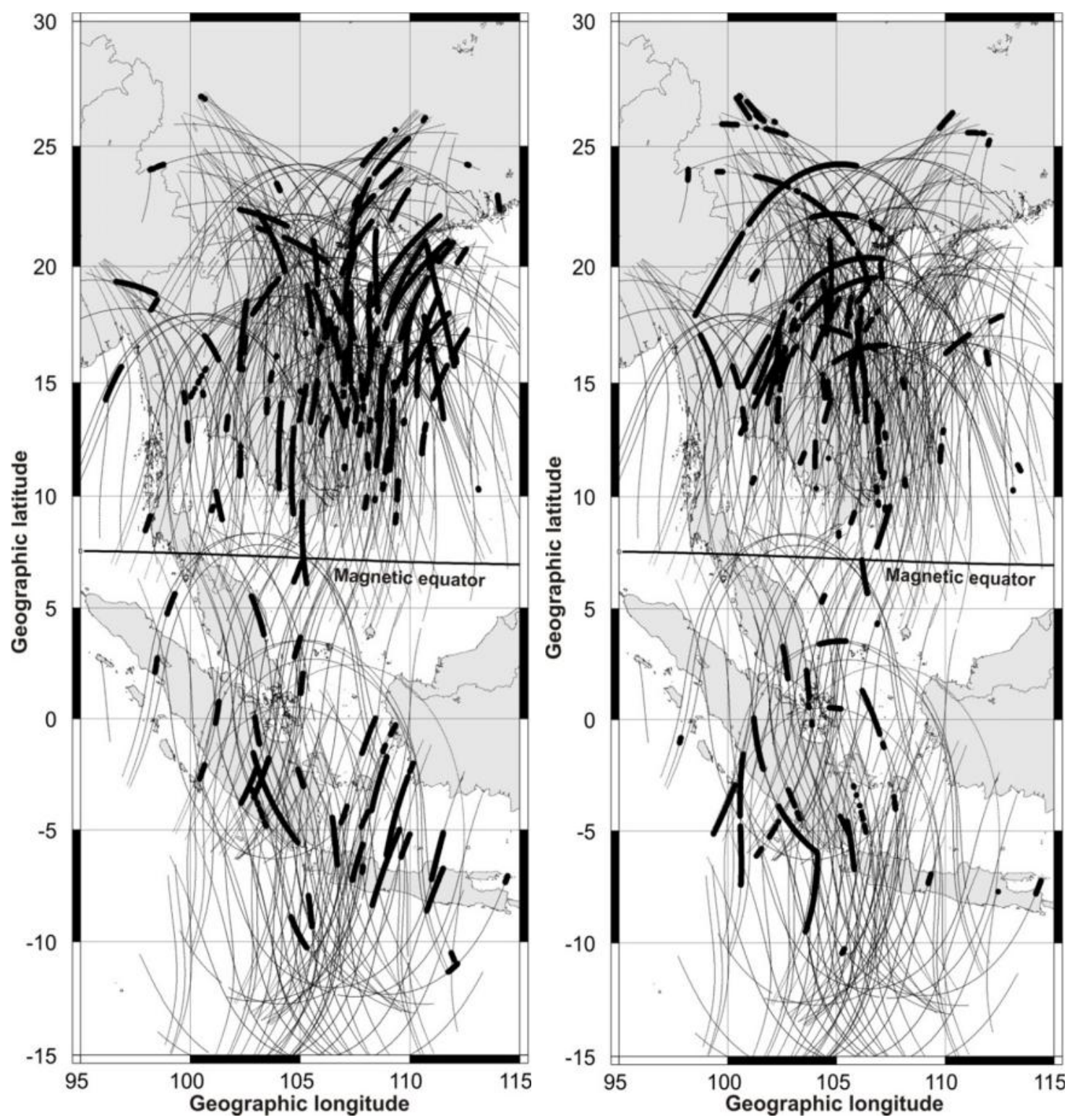

Figure 8. Geographic distribution of ROTI on a) 16 March 2015 and b) 19 March 2015 observed by 9 GPS stations in Southeast Asia. Black cycles: ROTI $\geq 0.5$; black small dots: ROTI $<0.5$ 
Vietnam Journal of Earth Sciences 38(3), 287-305
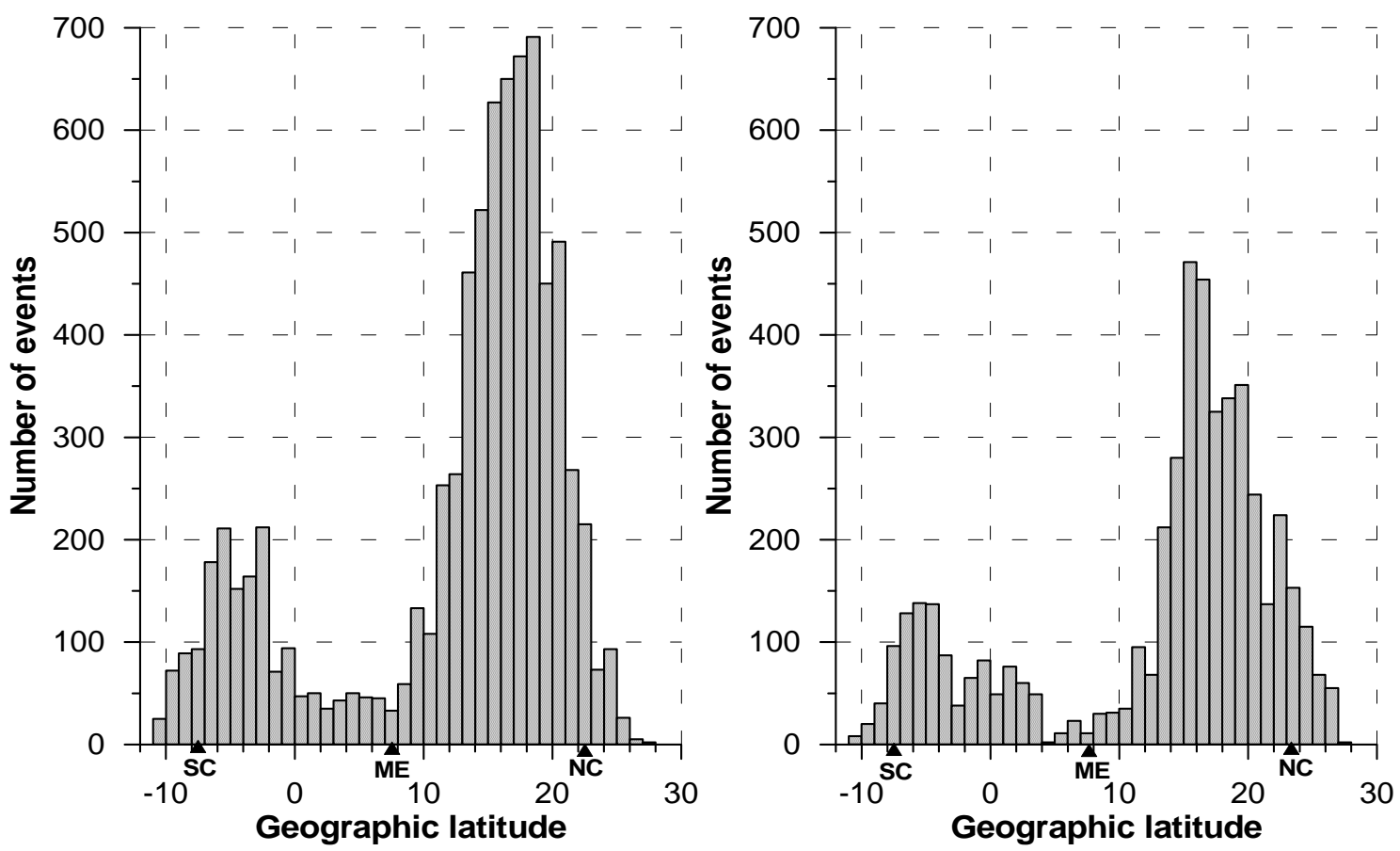

Figure 9. Statistics of scintillations ( $R O T I \geq 0.5$ ) along geographic latitude on 16 and 19 March 2015.ME: magnetic equator, SC: southern crest, NC: northern crest

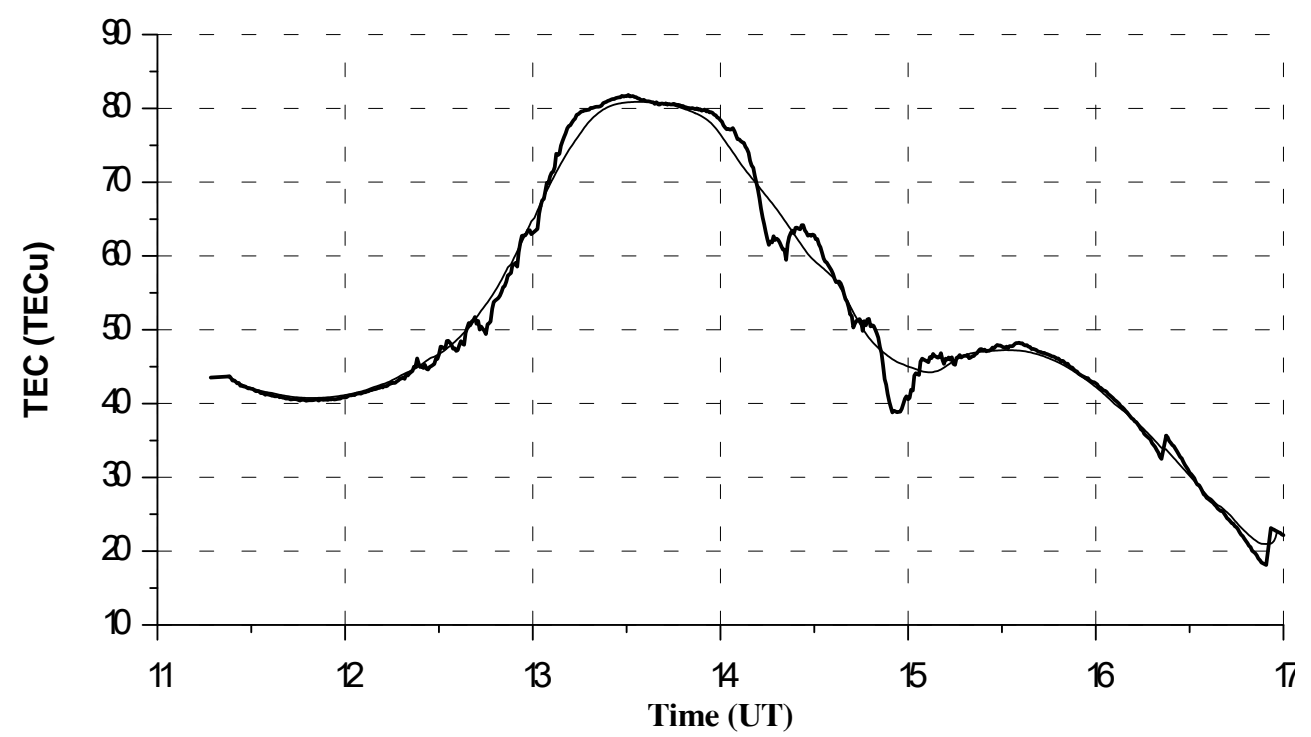

Figure 10. Total electron content computed from the PRN1-receiver pair at PhuThuy on the 16 March 2015 (solid line), and 30 minute running average (smooth thin line) 
L.H. Minh, et al./Vietnam Journal of Earth Sciences 38 (2016)

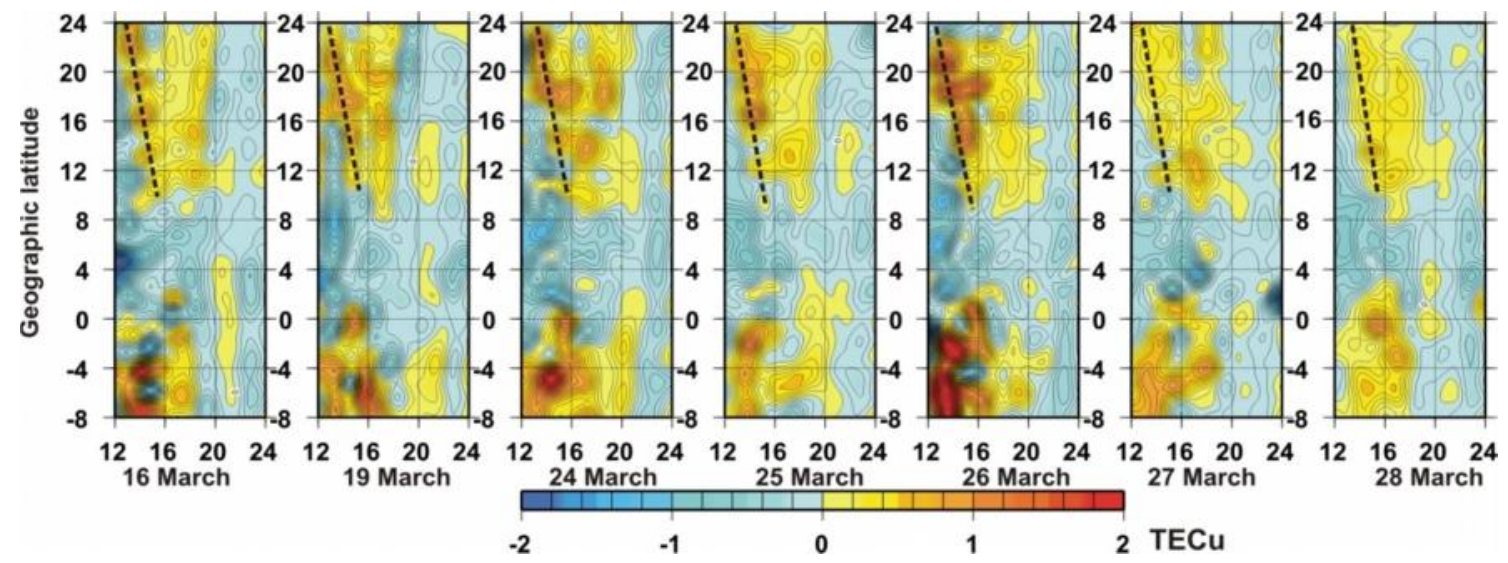

Figure 11. Time-latitudinal maps of TEC perturbation for the nights of the 16 March, 19 March, 24-28 March 2015 observed in the Southeast Asian region. Contour interval: $0.1 \mathrm{TECu}$

\section{Conclusions}

Based on the observations and calculations, we can draw some conclusions:

The results of TEC calculation by using the combination of the phase and pseudo range measurements are less dispersed than those by using only the pseudo range measurements.

The magnetic storm whose the main phase stated on 17 March 2015 was the big storm. The minimum values of the $\mathrm{SYM} / \mathrm{H}$ index of $223 \mathrm{nT}$ strongly affected the development of the equatorial ionization anomaly during the storm time. As observed in other storms, in the main phase, the anomaly crests had the increase in their amplitudes and expanded poleward, which was due to the direct penetration of the magnetospheric electric field into the equatorial ionosphere, increasing the fountain effect. On the first day of the recovery phase, owing to the influence of the ionospheric disturbance dynamo, the northern crest of the EIA experienced the degeneration in the amplitude and moved equatorward a long distance $\left(11^{\circ}\right)$, the southern crest completely disappeared. Such the variations is only observed in the heavy magnetic storms.
On the beginning day of the main phase, when the magnetic activity index was high, the ionospheric disturbances (scintillations) appeared sparsely; on the first day of the recovery phase, when the dynamo effect developed, the ionospheric disturbances were almost prevented. On some days in the storm time, when the magnetic activity index Ap was less than several tens of nT, the ionospheric disturbances appeared strongly. The ionospheric disturbances mainly appeared in the equatorial ionization anomaly region with the maximum appearance frequency being a few latitudes equatorward away from the anomaly crests.

The ionospheric disturbances (scintillations) observed in the storm are due to the mediumscale travelling ionospheric disturbances (MSTID) generated by acoustic-gravity waves in the northern crest region of the equatorial ionization anomaly after sunset moving equatorward with the velocity of about $210 \mathrm{~m} / \mathrm{s}$.

\section{Acknowledgements}

This article is finished thanks to the financial support of the VAST project VAST01.02/15-16. 
Vietnam Journal of Earth Sciences 38(3), 287-305

\section{References}

Abadi P., S. Saito, and W. Srigutomo, 2014. Low-latitude scintillation occurrences around the equatorial anomaly crest over Indonesia, Ann. Geophys., 32, 7-17.

Afraimovich E. L., 2008. First GPS-TEC evidence for the wave structure excited by the solar terminator, Earth, Planets and Space, 60, 895-900.

Afraimovich E. L., E. I. Astafyeva, V. V. Demyanov, I. K. Edemskiy, N. S. Gavrillyuk, A. B. Ishin, E. A. Kosogorov, L. A. Leonovich, O. S. Lesyuta, K. S. Palamartchouk, N. P. Perevalova, A. S. Polyakova, G. Y. Smolkov, S. V. Voeykov, Y. V. Yasyukevich and I. V. Zhivetiev, 2013. A review of GPS/GLONASS studies of the ionospheric response to natural and anthropogenic processes and phenomena, J. Space Weather Space Clim., 3, A27, DOI:10.1051/swsc/2013049.

Afraimovich E. L., I. K. Edemskiy, S. V. Voeykov, Yu. V. Yasyukevich and I. V. Zhivetiev, 2009. The first GPS-TEC imaging of the space structure of MS wave packets excited by the solar terminator, Ann. Geophys., 27, 1521-1525.

Appleton E., 1946. Two anomalies in the ionosphere, Nature, 157,691 .

Blanc M., A. Richmond, 1980. The ionospheric disturbance dynamo, J. Geophys. Res., 85, 1669-1686.

Borries C., N. Jakowski and V. Wilken, 2009. Storm induced large scale TIDs observed in GPS derived TEC, Ann. Geophys., 27, 1605-1612.

Budden K. G., 1985. The propagation of radio waves, Cambridge University Press, New York, 669p.

Carrano C. \& K. Groves, 2009. Ionospheric data processing and analysis. Workshop on Satellite Navigation Science and Technology for Africa, The Abdus Salam ICTP, Trieste, Italy.

Davis M. J., 1971. On polar substorms as the source of largescale traveling ionospheric disturbances, J. Geophys. Res., 76,4525 .

Davies K. and J. E. Jones, 1971. Three-dimensional observations of traveling ionospheric disturbances, J. Atmos. Terr. Phys., 33, 39.

Fejer B. G. and J. T. Emmert, 2003. Low-latitude ionospheric disturbance electric field effects during the recovery phase of the 19-21 October 1998 magnetic storm. J. Geophys. Res., 108. doi: 10.1029/2003JA010190.
Fejer B. G., C. A. Gonzales, D. T. Farley, M. C. Kelley and R. F. Woodman, 1979. Equatorial electric field during magnetically disturbed conditions: 1 . the effect of interplanetary magnetic field, J. Geophys. Res., 84, 5797.

Fejer B. G. \& L. Scherliess, 1995. Time dependent response of equatorial ionospheric electric fields to magnetospheric disturbances, Geophys. Res. Lett., 22, 851-854.

Fejer B. G., R. W. Spiro, R. A. Wolf and J. C. Foster, 1990. Latitudinal variation of perturbation electric fields during magnetically disturbed periods: 1986 SUNDIAL observations and model results, Ann. Geophys., 8, 441-454.

Foster J. C. \& F. J. Rich, 1998. Prompt mid-latitude electric field effects during severe geomagnetic storms, J. Geophys. Res., 103, 26367-26372.

Francis S. H., 1974. A theory of medium-scale traveling ionospheric disturbances, J. Geophys. Res., 79, 34, 5245-5260.

Georges T. M., 1968. HF Doppler studies of traveling ionospheric disturbances, J. Atmos. Terr. Phys., 30, 735.

Gonzales C. A., M. C. Kelley, B. G. Fejer, J. F. Vickrey and R. F. Woodman, 1979. Equatorial electric fields during magnetically disturbed conditions: 2. Implications of simultaneous auroral and equatorial measurements, J. Geophys. Res., 84, 5803.

Hines C. O., 1960. Internal atmospheric gravity waves at ionospheric heights, Can. J. Phys., 38, 1441.

Jaggi R. K. and R. A. Wolf, 1973. Self-consistent calculation of the motion of a sheet of ions in the magnetosphere, J. Geophys. Res., 78, 2852-2866.

Kelley M. C., B. G. Fejer and C. A. Gonzales, 1979. An explanation for anomalous equatorial ionospheric electric fields associated with a northward turning of the interplanetary magnetic field, Geophys. Res. Lett., 6, 301-304.

Kelley M. C., J. J. Makela, J. L. Chau, and M. J. Nicolls, 2003. Penetration of the solar wind electric field into the magnetosphere/ionosphere system, Geophys. Res. Lett., 30(4), 1158, doi:10.1029/2002GL016321.

J. Klobuchar, 1986. Design and characteristics of the GPS ionospheric time-delay algorithm for single frequency users, in: Proceedings of PLAN'86 -Position Location and Navigation Symposium, Las Vegas, Nevada, 280-286, 4-7, November. 
L.H. Minh, et al./Vietnam Journal of Earth Sciences 38 (2016)

Kikuchi T., H. Lühr, K. Schlegel, H. Tachihara, M. Shinohara and T. I. Kitamura, 2000. Penetration of auroral electric fields to the equator during a substorm, J. Geophys. Res., $105,23251-23261$

Fuller-Rowell T. M., G. H. Millward, A. D. Richmond and M V. Codrescu, 2002. Storm-time changes in the upper atmosphere at low latitudes, J. Atmos. Sol. Terr. Phys., 64, 1383.

Tran Thi Lan, Le Huy Minh, 2011. The temporal variations of the total electron content (TEC) and the ionospheric scintillation according to the continuous GPS data in Vietnam, Journal of Sciences of the Earth, 33(4), 681-689.

Tran Thi Lan, Le Huy Minh, R. Fleury, Tran Viet Phuong, Nguyen Ha Thanh, 2015. The occurrence characteristics of the ionospheric scintillation in Vietnam in the period 20092012, Journal of Sciences of the Earth, 37(3), 264-274 (in Vietnamese).

Le Huy Minh, C. Amory-Mazaudier, R. Fleury, A. Bourdillon, P. Lassudrie-Duchesne, Tran Thi Lan, Nguyen Chien Thang, Nguyen Ha Thanh, P. Vila, 2014. Time variations of the total electron content in the Southeast Asian equatorial ionization anomaly for the period 2006-2011, Advances in Space Research, 54, 355-368.

Lin C. H., A. D. Richmond, J. Y. Liu, H. C. Yeh, L. J. Paxton, G. Lu, H. F. Tsai, S. -Y. Su, 2005. Large-scale variations of the low-latitude ionosphere during the OctoberNovember 2003 superstorm: Observational results, J. Geophys. Res., 110, A09S28, doi:10.1029/2004JA010900.

Liu J. Y., H. F. Tsai and T. K. Jung, 1996. Total electron content obtained by using the global positioning system, Terr. Atmos. Oceanic Sci., 7, 107-117.

Ma G. and T. Maruyama, 2006. A super bubble detected by dense GPS network at east Asian longitudes, Geophys., Res. Lett., 33, L21103, doi:10.1029/2003JA009931.

Le Huy Minh, A. Bourdillon, P. L. Duschesne, R. Fleury, Nguyen Chien Thang, Tran Thi Lan, Ngo Van Quan, Le Truong Thanh, Tran Ngoc Nam, Hoang Thai Lan, 2006. The determination of the ionospheric total electron content in Vietnam from the data of GPS stations, Journal of Geology, A(296), 54-62.

Munro G. H., 1958: Travelling ionospheric disturbances in the F region, Aust. J. Phys., 11, 91.
Nishida A., 1968. Coherence of geomagnetic DP 2 fluctuations with interplanetary magnetic variations, J. Geophys. Res., 73(17), 5549.

Peymirat C. and D. Fontaine, 1994. Numerical simulation of magnetospheric convection including the effect of fieldaligned currents and electron precipitation, J. Geophys. Res., 99, 11155-11176.

Pi X., A. J. Mannucci, U. J. Lindqwister and C. M. Ho, 1997. Monitoring of global ionospheric irregularities using the worldwide GPS network, Geophysical Research Letters, 24(18), 2283-2286.

Richmond A. D., C. Peymirat, and R. G. Roble, 2003. Longlasting disturbances in the equatorial ionospheric electric field simulated with a coupled magnetosphere-ionospherethermosphere model, J. Geophys. Res., 108(A3), 1118, doi:10.1029/2002JA009758.

Spiro R. W., R. A. Wolf and B. G. Fejer, 1988. Penetration of high-latitude-electric-field effects to low latitudes during SUNDIAL 1984, Ann. Geophys., 6, 39-50.

Saito A., S. Fukao and S. Miyazaki, 1998: High resolution mapping of TEC perturbations with the GSI GPS network over Japan, Geophys. Res. Lett., 25, 3079-3082.

Sastri J. H., 1988. Equatorial electric fields of ionospheric disturbance dynamo origin, Ann. Geophys., 6(6), 635-642.

Shimeis A., C. Borries, C. Amory-Mazaudier, R. Fleury, A. M. Mahrous, A. F. Hassan, S. Nawar, 2015. TEC variations along an East Euro-African chain during $5^{\text {th }}$ April 2010 geomagnetic storm, Advances in Space Research, 55, 2239-2247.

Shiokawa K., Y. Otsuka, M. K. Ejiri, Y. Sahai, T. Kadota, C. Ihara, T. Ogawa, K. Igarashi, S. Miyazaki and A. Saito, 2002. Imaging observations of the equatorward limit of midlatitude traveling ionospheric disturbances, Earth, Planets and Space, 54, 57-62.

Spogli L., L. Alfonsi, G. De Franceschi, V. Romano, M. H. O. Aquino, A. Dodson, 2009. Climatology of GPS ionospheric scintillations over high and mid-latitude European regions, Ann. Geophys., 27, 3429-3437.

Van Dierendonck A. J., J. Klobuchar, Quyen Hua, 1993. Ionospheric scintillation monitoring using commercial single frequency C/A code receivers, Proceedings of ION GPS-93. 
Vietnam Journal of Earth Sciences 38(3), 287-305

Vasyliunas V. M., 1970. Mathematical models of magnetospheric convection and its coupling to the ionosphere, in Particles and Fields in the Magnetosphere, edited by McCormac, 60-71, Springer, New York.

Vasyliunas V. M., 1972. The interrelationship of magnetospheric processes, in Earth's Magnetospheric Processes, edited by McCormac, 29-38, Springer, New York.
Tsugawa T., Y. Otsuka, A. J. Coster and A. Saito, 2007. Medium-scale traveling ionospheric disturbances detected with dense and wide TEC maps over North America, Geophys. Res. Lett., 34, L22101, doi:10.1029/2007GL031663.

Zhao B., W. Wan, L. Liu, Z. Ren, 2009. Characteristics of the ionospheric total electron content of the equatorial ionization anomaly in the Asian-Australian region during 1996-2004, Ann. Geophys., 27, 3861-3873. 